\title{
Computerized Cell Image Analysis: Past, Present, and Future
}

\author{
Invited \\ Ewert Bengtsson \\ Centre for Image Analysis, Uppsala University \\ Lägerhyddvägen 3, 75237 Uppsala, Sweden \\ ewert@cb.uu.se
}

\begin{abstract}
The visual interpretation of images is at the core of most medical diagnostic procedures and the final decision for many diseases is based on microscopic examination of cells and tissues. Through screening of cell samples the incidence and mortality of cervical cancer have been reduced significantly. The visual interpretation is, however, tedious and in many cases error-prone. Therefore many attempts have been made at using the computer to supplement or replace the human visual inspection by computer analysis and to automate some of the more tedious visual screening tasks. In this paper these developments are traced from their very beginning through the present situation and into the future. The paper concludes with a discussion of the possibilities of applying the gained experiences to create the toolbox needed to turn functional genomics from prospect to reality.
\end{abstract}

\section{Introduction}

As digital images are becoming ubiquitous and image processing is taking place in standard and embedded computers everywhere it is time to briefly look back at the history of computerized image analysis and see what we have learned.

One of the first and largest application fields for image analysis is in medicine and especially so in cytology. This should be no surprise since the visual interpretation of images is at the core of most medical diagnostic procedures. The final diagnostic decision for many diseases is based on microscopic examination of cells and tissues. In particular, cancer is always finally diagnosed through the microscope. In addition to the diagnosis, the microscopic examination can help in grading the cancer, i.e. to determine how aggressive it is and how it should be treated. In addition, if cell samples can be examined from healthy individuals, precursors of cancer can be detected even before they develop into the dangerous disease and often cured by simple means. The most prominent example is the screening for cervical cancer through the so called Pap-smear, which has reduced the incidence and mortality of the second most common cancer for women significantly in countries with an effective screening program. The visual interpretation is, however, tedious and in many cases error-prone. Therefore, ever since the first appearance of computers, significant development efforts have been aiming at supplementing or replacing the human visual inspection by computer analysis.

With an over-simplification we can divide the computer usage motivations into on one hand the efforts at automating the tedious visual analysis of the massive numbers 
of specimens originating from mass screening programs such as the screening for cervical cancer. On the other hand we have the quantitative measurement of some significant aspect of the cell e.g. the amount of DNA per cell nucleus. In this presentation we will examine these two approaches, follow their historical development and try to see to what extent they are addressing the same problems. We will also examine where we stand today and try to say a few words about where cell image analysis technology will take us in the future.

\section{The Past}

\section{The beginning}

Understanding the cell. In the thirties Caspersson at the Karolinska Institute discovered that it was possible to measure the nucleic acids of cells with photometric techniques [1]. He used UV absorption measurements to determine the amount of cellular constituents such as DNA and RNA. The invention of the Feulgen reaction made this methodology accessible to many more laboratories and in the fifties much research was carried out that established quantitative microphotometry as a useful scientific discipline. The hope was that the straightforward measurement of the amount of DNA per nucleus would distinguish abnormal and normal cells. Unfortunately this was found not to be true. Considerable variation in the amount of DNA per cell was found both in normal cells and in tumor cells. Many conflicting reports were published and no real consensus concerning the value of cell DNA measurements could be reached.

Early cervical cancer pre-screening. Screening for cervical cancer has been done since Papanicolaou and Traut in 1943 in a monograph described in detail how to identify cancer cells by examining a stained cell specimen in a light microscope [2]. The use of screening has led to more cancers being detected in an early stage, and this in turn has led to a dramatic reduction in the death rate for women who have been tested regularly $[3,4]$. The screening programs, however, generate enormous numbers of samples to process, currently about 160 million samples per year. And this should increase by a factor of 10 if all women were to be given reasonable protection against developing undetected cervical cancers. Educating and financing sufficient numbers of human screeners early seemed unfeasible and this created an interest in the possibilities of automating the task.

Automation. The first ideas about an automatic device for cancer cell screening appeared in the fifties [5]. The proposed machine was based on the concept that cancer cells could be distinguished from normal cells on the basis of nuclear size and optical density. A multi-million dollar project was launched and the first fully automated microscope system, the Cytoanalyzer was built. This system included automatic slide feed and auto-focus circuits. The image analysis was based on hardwired analogue video processing circuits that generated two-dimensional histograms of nuclear size versus nuclear optical density. The spatial resolution was not very high, 2 - 10 micrometers [6]. Tests with the Cytoanalyzer revealed that the special purpose fixed logic pattern recognition computer was both expensive and 
ineffective [7]. The project failed in the early sixties mainly because the system was unable to detect the artifacts in the sample preparations i.e. clumps of blood cells, strands of tissue and mucus, overlapping epithelia cells etc.

Due to the bad reputation for cytology automation caused by this early failure in the US the attempts at automation over the next couple of decades were shifted to Europe and Japan. One such early project was the one-parameter (nuclear size) automatic screener developed in Britain in the late sixties [8]. In Japan Watanabe and co-workers at Toshiba developed CYBEST [9]. Their first version used special purpose electronic circuits while later versions were based on general purpose digital computers, thus bridging the gap between old and new technology. But none of these projects were successful.

The first generation interactive cell image analysis systems The failure of the early attempts at automation based on special purpose signal processing circuits and the failure of tumor cell characterization based on single cell measurements both led to the need for more flexible and powerful image analysis systems. The breakthrough for general purpose computers in the early sixties made the design and implementation of such systems possible, although still difficult since the typical computer memories were limited to tens of kilobytes. One of the first was CYDAC [10], which was based on the Cytoanalyzer. Other systems were TICAS in Chicago [11], LEYTAS in the Netherlands [12], SAMBA in France [13] and CELLO at our laboratory [14].

These systems were used for the exploration of cell image analysis algorithms, both with the goal of achieving fully automated cervical prescreening and more knowledge about the differences between various cell types through more detailed quantitation of cell morphology and sub-cellular patterns. The results were published in hundreds of scientific papers. And the optimism concerning the usefulness of quantitation and feasibility of automation grew.

\section{Second generation systems}

Automated cytology Around 1980, various research group were again ready to start building prototypes of fully automated devices. Systems developed were BioPEPR [15], FAZYTAN [16], CERVISCAN [17], LEYTAS [18] and at our laboratory the DIASCANNER [19]. Most of these systems reached an operational prototype stage in the mid eighties. Some reported classification accuracies that were well within the range of what is achieved by the conventional visual screening. But none of the systems reached the market. The projects seemed to stumble on the goal line.

Quantitative cytology The disappointment caused by the failure of simple integrated cell measurements caused some researchers in the early seventies to use the more powerful image analysis systems that then became available for more detailed measurements on the cell images, e.g. measurement of nuclear texture and morphology. Various measures of these kinds of features were shown to provide highly discriminatory information between normal and abnormal cells. But the measurements were difficult to make in a reliable way in routine work since they are taken at the limit of optical resolution and thus greatly affected by focus and 
preparation artefacts. Many studies also used questionable statistical data analysis methods, using too many feature candidates and powerful multivariate analysis on materials with few cases, thus creating results that could not be reproduced by other researchers [20]. So in practice these more sophisticated measurements had limited impact on mainstream cytology and pathology.

Eventually more careful work on the interpretation of the distribution of the measurements of amount of DNA per cell found in a cell population, the so called ploidy pattern, revealed significant differences between normal cell populations and tumour cell populations. Moreover it turned out that the ploidy pattern also could be used to discriminate between tumours of low versus high malignancy. Several studies showed clear correlations between ploidy patterns and patient prognosis [21]. The determination of the ploidy pattern required a whole population of cell nuclei to be measured something that was quite tedious with the first generation slow cytophotometers. Thus the higher speed instruments originally intended for automated cytology also found use in the quantitative research line.

\section{Third generation systems}

Automated prescreeners. In the late eighties there was a great increase in interest in cytology automation in the US for economic/legal reasons and many new projects were started [22]. One new aspect that appeared at this time was new ways of preparing the samples. The Cytyc corporation had developed their own automated specimen preparation technique, ThinPrep, that made much cleaner specimens than the conventional smears, at the expense of significantly more complex preparation technique [23]. Another similar preparation method was developed by AutoCyte [24].

The PAPNET system from Neuromedical Systems used conventionally prepared Pap-smears [25]. After an initial low resolution object search, high resolution fields were processed first by an algorithmic classifier and then by a neural network classifier. This was one of the first large scale uses of neural networks in a commercial image analysis based product. The output of the classifiers was a ranking of the abnormality of the detected "cells" so that images of the 64 most abnormal ones could be stored on a magnetic tape and later shown to the cytotechnologist at a review station where the final decision whether the specimen should be classified as normal or suspicious was taken. PAPNET thus introduced interaction into automated prescreening.

The AutoPap 300 from NeoPath was similar to PAPNET in that it uses conventional Pap-smears and neural network classifiers [26]. It increased the image acquisition rate by utilizing strobed illumination of a CCD sensor thus allowing very rapid search of the specimen. This was used at two resolution levels, an initial low resolution mapping of the specimen, followed by a high resolution field by field analysis of the most "interesting looking" parts of the specimen. The image processing was carried out in custom designed processing boards of which up to ten could be connected in parallel to increase processing power. Most of the processing was based on mathematical morphology operations resulting in as many as 68 different features being sent to the classifiers. The final result was a "normal" $v s$ "requires visual inspection" decision on the specimen level, i.e. no interactive confirmation was used of the machine decision for the negative cases. 
In another project AccuMed International in Chicago acquired the rights to the Uppsala Diascanner system and built a screening system based on dual resolution scanning and analysis steps.

Quantitative Cytology Systems The general computer development continued and in the late eighties a typical interactive image analysis system consisted of a standard computer equipped with a video frame grabber and color image display, sometimes also a dedicated image processor, many such systems were developed and a few became products.

The main clinical use for these systems was DNA ploidy analysis. The acceptance of these analyses in routine health care varied between different countries. In Holland where the health care authorities were prepared to pay for the cytologic quantification over the health insurance these devices could be found in the great majority of pathology laboratories. In other countries DNA ploidy analysis was still only used mainly or exclusively for research purposes.

Many systems were also used for other research tasks quantifying various other cellular markers than DNA. Any biochemical reaction that can cause a cell or cellular component to change colour or appearance in some way can be measured. In particular, various immunostaining techniques that stained selected proteins or other cellular constituents in a highly specific way were developed. In this application the superior capability of the computer to count and measure the number of stained objects and their area was of great value [27,28].

Tissue level analysis. Another topic of interest was the organization of the tissue, sometimes called cellular sociology. When a pathologist is grading a cancer specimen, i.e. trying to judge how invasive the tumor will be, he is judging the degree to which the cancer has caused the natural order and structure in the tissue to break down. Finding all the cells in a tissue sample and then describing their relative distances, orientations etc through graph analysis, the computer can generate grading that is similar to that of the pathologist but in a more quantitative and hopefully more objective and reproducible way [29,30]

A similar question is how good the tumour is at generating new vascular structures. Without a functioning vascular network the tumour can not grow beyond a certain size due to lack of supplies which the blood stream provides. Pathologists have therefore become interested in determining the degree of vascularisation in tumour tissue. Again, computerized image analysis can do this in a more objective way than visual inspection alone [31].

\section{Computer acceptance by pathologists.}

For a long time one of the obstacles in getting computerized image analysis accepted in routine clinical pathology was the limited experience of using computers among pathologists and other medical professionals. Having a computerized system in the lab was an alien creature requiring specialized support functions etc. Pathologists were used to look in microscopes, not on computer screens. 
Highly Optimized Microscope Environment. One attempt at dealing with this issue was the HOME (Highly Optimized Microscope Environment) project in the early nineties [35]. The key feature in this approach was to integrate the computer display in the optical path of the microscope. Thus the pathologist looked down the microscope as usual and saw a computer generated display superimposed on the microscope field. The only input device was a mouse controlled pointer that could be used to point at menu buttons or cells. In its simplest form this could be used for tagging, counting and bookkeeping in visual image analysis. The areas and shapes of regions interactively outlined could also be measured. But the concept could also be extended with a computer image analysis system controlled directly from the microscope making it possible to carry out any quantitative image analysis task. Since this concepts removed many of the "strange" computer components it was hoped that it would be easier to accept than the more conventional CRT-display based approaches. But the ergonomic issues were problematic and the project did not have any major impact.

\section{The present}

\section{The present situation in cervical pre-screening}

During the nineties there was strong competition between the American companies developing screening technology as well as struggles to get the various solutions approved by the powerful Food and Drug Administration, FDA. No system can be sold in the US without FDA approval. Hundreds of millions were spent on developments and field trials and in the end only one automatic image analysis based screening system vendor remained.

That vendor is Tripath, which is the merger of NeoPath, Neuromedical and AutoCyte. They are selling a system, called Focal Point, which to a large extent is based on the AutoPap 300 [32]. It does a good job at recognizing clearly normal specimens while the suspicious ones need human inspection. In the US that second, visual screening has to be done completely manually, due to lack of FDA approval, while in many other parts of the world the human screener can use a computer controlled microscope that shows all the suspicious cells and their neighbourhoods. The system is selling for routine use, so finally after some 50 years of research there is a working system for computer supported Pap-smear screening on the market.

Cytyc has been quite successful with their improved preparation technique and can demonstrate better performance for that technique as compared to conventional smears. Their specimens can also be used in automatic systems but they are not themselves actively marketing any such system [33]

Molecular Diagnostics took over AccuMed and they are currently developing a completely new approach to screening based on highly specific genetic probes that react with the known genetic changes associated with cancer. From an image processing point of view it mainly becomes a matter of discovering a strongly deviating colour for the positive cells. If they are successful it may very well change the whole way cervical cancer is screened and detected [34]. 


\section{Telepathology}

As the general computer revolution has moved on computers have become commonplace also in pathologist offices. They are used for normal office work and for email. A natural extension of email is to send difficult images to fellow pathologist to get their second opinion. Thus the pathologists have a new motivation for having a digital camera attached to his microscope. Telepathology, as this application is called, has also been developed in more organized and commercial ways. But its net effect has been to make the basic infrastructure for computer assisted cell image analysis more natural and readily available for pathologists. This is paving the way for a broader usage of the technologies, although the impact on the everyday practices of pathology are still lower than many of us active in the field would have predicted.

\section{Cytomics}

This paper has so far mainly been dealing with cancer related topics. And it is true that cell image analysis for the first 50 years has been heavily oriented towards cancer issues. Not completely though, there have been very interesting developments of automated blood cell differential counters with a history about as long and turbulent as that of the cancer screening machines. And similarly there have been substantial developments in the field of computer assisted chromosome analysis systems. But those will have to be left outside this paper.

Today, however, the rapid development in modern bioscience is opening a whole new application field for the tools that we have been developing for cancer cell image analysis over the last decades.

The well known HUGO project led to an almost complete map of the human genome and currently significant efforts are focused on mapping what proteins the different genes are expressing. In order to completely understand the functioning of the cells and organs of the body it is also necessary to map when and where the different genes are activated.

Cytomics is the science of cell-based analysis that integrates genomics and proteomics with the dynamic function of cells and tissues. Many recognize functional genomics, or cytomics as the next great challenge for bioscience. Most of the previous work on mapping genes and protein expressions has been done in bulk, without the possibility of linking the events to specific positions and functions in the cells. In order to move on to deeper understanding of the cell development and behaviors, new microscopy methods will be at the core of the developments. There has also been substantial progress in the development of new microscopy techniques. One of the April 2003 issues of the Science journal which had biological imaging as the main theme gives a good overview of this [36]. And in May 2003 the first international Cytomics conference took place in Wales [37].

A seriously limiting factor in microscopy is the human observer. Here the same factors as we have previously discussed in relation to cancer cell image analysis applies. Humans are very good at recognizing and interpreting patterns qualitatively but we are very poor at quantitative evaluations. Also viewing each microscopic field visually takes time. Whereas the techniques developed for discovery of genes and proteins in bulk solutions can process thousands of substances in parallel the current 
microscopy techniques are limited to a few substances. In the study of cell development the cells have to be followed for long periods of time making visual interpretations highly impractical. All these factors make automatic, or computer assisted, image analysis methods necessary in order to facilitate continued progress in the understanding of cell dynamics. This makes quantitative image cytomics a key area in the future development of bioscience. There are groups recognizing this and in one interesting project, The Open Microscopy Environment, several groups have joined forces to create an open site and software standard for exchanging well documented image data sets and extracted features that can be used for evaluation of new algorithms as well as new hypotheses about cell functional patterns [38].

One of the main goals of functional genomics, proteomics and cytomics is to understand how the cells develop and differentiate from stem cells into the highly specialized organ cells, to understand how and when the genes are expressed in the cell and how the signaling inside and between cells works. We thus need to study the cells on several levels of resolution. Let's outline a few current examples.

Time-Lapse Microscopy and Cell Migration Modelling. In order to study and control stem cell multi-potency and self-renewal the cell migration and cell division have to be modeled. This is done by tracking individual cells over time. Image sequences are acquired using time-lapse microscopy supplemented by an incubator system to keep the cells alive and a motorized stage to make it possible to investigate and compare multiple cell cultures, e.g. manipulated cells and controls. Tracking the cells in these sequences presents a number of image analysis challenges because cells may be touching and overlapping as well as entering or leaving the microscopic field of view. The robustness and accuracy of automated tracking methods can be improved if statistical a priori information on typical cell movement patterns can be incorporated into the tracking algorithm.

A time-lapse microscopy and cell tracking system has recently been developed in Göteborg [39]. The team has also in a preliminary study tried Hidden Markov Modeling (HMM) of the cell motion pattern [40]. The system is being used in the Institute of Clinical Neuroscience, in a series of experiments on stem cells and for modeling neutrophil chemotaxis.

Analysis of Sub-Cellular Compartments and Transportation. Cells are elaborately subdivided into functionally distinct, membrane-bounded compartments. Each compartment contains its own characteristic set of specialized molecules, and complex distribution systems transport specific products from one compartment to another or from one compartment and out of the cell. Specific proteins catalyze reactions within compartments and selectively transport molecules between compartments. Signals from outside the cell can also trigger specific transport within, as well as into and out of, the cell, e.g. resulting in the secretion of insulin from cells of the pancreas.

By tracking the protein traffic from one compartment to another, a better understanding of the maze of intracellular membranes and signals can be achieved. Specific proteins within living cells can be tagged with GFP, green fluorescent protein, and similar tags, by genetic engineering. The localization and movement of 
the tagged protein can thereafter be imaged using fluorescence microscopy. Corresponding studies on other types of tagged biomolecules, such as metabolites, can also be performed. Specific intracellular compartments and structures can be visualized by immunofluorescence staining. The analysis of these signals can often be described as the analysis of fluorescent "blobs". Methods for detection, characterization, quantification and positioning of blobs are needed. This includes the needs for specialized segmentation methods as well as methods for tracking and extraction of features describing morphology. In addition to the use in basic bioscience research these kinds of methods are also of great interest to the pharmaceutical industry in the development of new drugs. In Uppsala we have developed methods for high speed analysis of so-called RAC-formation in collaboration with Amersham Biosciences [41,42].

Highly specific molecular probes and co-localisation studies. Several biotechnology research groups have developed highly specific high resolution stains. For instance Mats Nilsson, and co-workers at the Department of Genetics and Pathology at The Rudbeck Laboratory, Uppsala University, are developing molecular analytic tools that permit studies of individual DNA, RNA, and protein molecules in situ at single-nucleotide resolution (nucleic acids) and with the ability of analyzing post-translational modifications and protein-protein interactions [43,44]. One major advantage with their probing strategy, apart from the ability of obtaining localized detection of individual macromolecules within cells, is that multiple probes can be added, specific for thousands of different targets with very little tendency for interference between the different probes. In order to take advantage of this staining capability matching image analysis methods need to be developed. Conventional optical filters are not sufficiently narrow and sharp to prevent crosstalk if more than a few channels are studied simultaneously. This calls for multi- or hyperspectral image analysis approaches similar to those used in modern remote sensing.

\section{The future}

So far my presentation has been based on scientific results as found in literature and on my own experience. Moving on to say something about the future will by necessity make the statements more speculative and open to other opinions. If someone at a future SCIA conference looks back at this presentation they will most likely find some of the statements in this section to be wrong. Still, let's bring out the crystal ball and look into the future of cell image analysis. One way of doing this is to re-examine the trends I have already described and try to see where they may bring us.

\section{In clinical pathology}

Telepathology is growing in several ways. Pathologists are becoming accustomed to registering digital images and sharing them with colleagues. It will be increasingly common that image sets of difficult cases are sent to international experts for second opinion. Those experts may very well partly rely on computerized image analysis to evaluate the samples. The referring pathologist does not really need to know to what extent he is consulting a human or computerized expert. 
The HOME concept of putting a computer inside the microscope will most likely be revived as the cost of CCD cameras, computers and displays become small as compared to the optics of the microscopes. The microscope will be capable of helping the pathologist not only in administrating the samples and remembering interesting fields but also in quantitative assessment of interesting objects.

We will thus see increasing computer support in the decision making process of the pathologists. But the final diagnostic decisions will for a long time rest with the human expert.

\section{In automation}

The automated Pap-screening systems that finally have made it to the market will become more generally accepted and cost effective and step by step will the purely visual screening be replaced by mixed modes with increasing degrees of machine analysis. New stains based on specific molecular probes will supplement the traditional morphological stains increasing the utility of the screening. Let's hope that these developments also will make effective screening available in countries where today it is too expensive.

\section{Cameras examining the body from the inside}

New high resolution imaging methods may even make it possible to analyse the cellular status of the cervical transition zone in situ without having to take out a cell sample, providing an immediate answer to the woman. Other new endoscopic imaging techniques will make it possible to inspect the respiratory or digestive systems at high resolution discovering early cellular changes in time to prevent development into cancer. In a longer perspective even more miniaturized robot camera systems may be sent into the body to investigate suspicious sites for the presence of precancerous cells

\section{New clinical imaging methods}

Improvements in tomographic imaging devices will bring the resolution to the micron levels making it possible to study tissues with cellular resolutions in situ in noninvasive ways. This is already becoming possible in small laboratory animals. Exploitation of new parts of the electromagnetic spectrum and new ways of reconstructing images will make an ever richer set of anatomic and functional $3 \mathrm{D}$ image data from the body available for analysis. Eventually we may have an automated system that scans the entire body at varying resolution zooming in on anything that looks suspicious and comes up with a clear answer about what is wrong and what needs to be done to cure the problem.

\section{The crucial tools for functional genomics}

The tremendous progress in bioscience with the mapping of the human genome as one of the most spectacular highlights has brought great hope of an understanding of the way the body functions to such an extent that a cure can be found for most of the 
major diseases. It is, however, as already described, not enough to have a list of genes and proteins, it is also necessary to know when and where they are activated and controlling the development of cells and organs and the functioning of the body. To study this, imaging with sufficient resolution in space, time and spectrally is needed. The billions of images that will be produced can only be analysed through automated and quantitative image analysis methods. Such methods will be developed and will be crucial in making continued progress in the bioscience revolution possible. Quantitative image cytomics will bring functional genomics from promise to reality.

\section{The prospect for cell image analysis}

If even only part of these predictions and prospects are possible they are good reasons why cell image analysis also in the future should be a most important area of image analysis research and of scientific research in general.

\section{Acknowledgements}

Over the three decades of research outlined in this presentation I have collaborated closely with several dozen colleagues in image analysis as well as in various biological and medical specialities. Without that collaboration the research would not have been possible. Rather than trying to list all colleagues here I refer to the reference list for the sited joint papers and say to all a general Thank you!

\section{References}

1. Caspersson T: Die Untersuchung der Nukleinsuerferteilung im Zellkern. Z Wiss Mikrosk Technik 53:403, 1936.

2. Papanicolaou GN \& Traut HF: Diagnosis of Uterine Cancer by the Vaginal Smear. Oxford University Press, New York, 1943.

3. Stenkvist B, Bergstrm R, Eklund G \& Fox C: Papanicolaou Smear Screening and Cervical Cancer. What Can You Expect? JAMA 252:1423-1426,1984

4. Christopherson WM, Parker JE, Mendez WM \& Lundin FE: Cervix Cancer Rates and Mass Cytologic Screening. Cancer 26:808-811, 1970.

5. Mellor RC \& Silver R: A microfluorometric scanner for the differential detection of cells: application to exfoliate cytology. Science 114: 356-360, 1951.

6. Tolles WE \& Bostrom RC: Automatic Screening of Cytological Smears for Cancer: The Instrumentation. Ann NY Acad Sci 63:1211-1218, 1956.

7. Spencer CC \& Bostrom RC: Performance of the Cytoanalyzer in Recent Clinical Trials. Jour Nat Cancer Inst 29:267, 1962.

8. Spriggs AI, Diamond RA \& Meyer EW: A New Principle for AutomatedScreening of Cervical Smears. Lancet 1:359, 1968.

9. Watanabe S and the CYBEST group: An Automated Apparatus for Cancer Prescreening: CYBEST. Comp Graph Image Proc 3:350-358, 1974

10.Bostrom RC \& Holcomb WG: CYDAC - A Digital Scanning Cytophotometer.IEEE Int Conv Rec 9:110-119, 1963

11.Wied GL, Bartels PH, Bahr GF \& Oldfield DB: Taxonomic Intra-cellularAnalytic System (TICAS) for Cell Identification. Acta Cytol 12:180-204,1968

12.Vrolijk J, Pearson PL \& Ploem JS: LEYTAS: A System for the Processing of Microscopic Images. Analyt Quant Cytol 2:41-48, 1980 
13.Brugal G, Garbay C, Giroud F \& Adelh D: A Double Scanning Microphotometer for Image Analysis: Hardware, Software and Biomedical Applications. J Histochem Cytochem 27:144-153, 1979.

14.Bengtsson E, Eriksson O, Jarkrans T, Nordin B \& Stenkvist B: CELLO - An Interactive System for Image Analysis. In Bolc L \& Kulpa L (eds.): Digital Image Processing Systems, Lecture Notes in Computer Science 109. Springer Verlag, Berlin, 1981.

15.Zahniser DJ: Automation of Pap Smear Analysis: A Review and Status Report. In Haralick, RM (ed.): Pictorial Data Analysis. Springer, 1983

16.Erhardt R, Reinhardt E, Schlipf W \& Bloss WH: FAZYTAN: A System for Fast Automated Cell Segmentation, Cell Image Analysis and Feature Extraction Based on TV-image Pickup and Parallel Processing. Analyt Quant Cytol 2:25-40, 1980.

17.Tucker JH \& Husain OAN: Trials with the CERVISCAN Experimental Prescreening Device on Polylysine-prepared Slides. Analyt Quant Cytol3:117-120, 1981.

18.Ploem JS, van Driel-Kulker AMJ \& Verwoerd NP: LEYTAS - A Cytology Screening System Using the New Modular Image Analysis Computer (MIAC) from Leitz. In Burger G, Ploem JS \& Goerttler K (eds.): Clinical Cytometry and Histometry: 24-35, Academic Press, London, 1987.

19. Nordin B: The Development of an Automated Prescreener for the Early Detection of Cervical Cancer. Algorithms and Implementation. PhD Thesis, Uppsala Univ., 155 p,1989.

20.Schulerud H,. Kristensen G.B,. Liestøl K, Vlatkovic L., Reith A, Albregtsen., F. Danielsen H.E.. A review of caveats in statistical nuclear image analysis, Analytical Cellular Path., 16: 63-82, 1998.

21.Fallenius A, Franzén S \& Auer G: Predictive value of nuclear DNA content in breast cancer in relation to clinical and morphologic factors. A retrospective study of 227 consecutive cases. Cancer 62: 521-530, 1988

22.Koss L G: The Papanicolaou Test for Cervical Cancer Detection - A Triumph and a Tragedy. JAMA 261:737-743, 1989.

23.Hutchinson ML, Cassin CM \& Ball HG: The Efficacy of an Automated Preparation Device for Cervical Cytology. Am J. Clinical Path. 96:300-305, 1991.

24.Howell LP, Davis RL, Belk TI, Agdigos R, Lowe J. The AutoCyte preparation system for gynaecologic cytology. Acta Cytol., 42:171-177, 1998.

25.DeCresce RP \& Lifshitz MS: PAPNET Cytological Screening System. Lab Medicine 22: 276-280, 1991.

26.Lee J, Nelson A, Wilbur DC, Patten SF. The Development of an Automated Papanicolau Smear Screening System. Cancer, 81: 332-336, 1998.

27.Ranefall P, Wester K, Bengtsson E. Automatic quantification of immunohistochemically stained cell nuclei using unsupervised image analysis.. Analytical Cellular Path., 58, 1997.

28.Ranefall P, Wester K, Andersson AC, Busch C, Bengtsson E. Automatic quantification of immunohistochemically stained cell nuclei based on standard reference cells. Analytical Cellular Path., 111-123, 1998.

29.Jarkrans T, Vasko J, Bengtsson E, Choi HK, Malmström PU, Wester K, Busch C. Grading of transitional cell bladder carcinoma by image analysis of histological sections: Analytical Cellular Path.. 8:135-158, 1995.

30.Choi HK, Vasko J, Bengtsson E, Jarkrans T, Malmström PU, Wester K, Busch C. Grading of Transitional Cell Bladder Carcinoma by Texture Analysis of Histological Sections.: Analytical Cellular Path. 6:327-343, 1994.

31.Ranefall P, Bengtsson E, Busch C, Malmström PU. Automatic Quantification of Microvessel Density in Urinary Bladder Carcinoma.. British Journal of Cancer, 81,8: 1363$1370,1999$.

32.Tripath website: http://www.tripathimaging.com/

33.Cytyc website: http://www.cytyc.com/

34.Molecular Diagnostics website: http://www.molecular-dx.com/ 
35.Brugal G., Dye R., Krief B., Chassery J.M., Tanke H., Tucker J.H. HOME: Highly Optimized Microscope Environment. Cytometry, Vol 13, pp 109-116, 1992.

36.SCIENCE Volume 300, Number 5616, Issue of 4 Apr 2003.

37.First International Cytomics Conference website: http://www.isac-net.org/cytomics/

38.The Open Microscopy Environment website: http://www.openmicroscopy.org/

39.Gustavsson T., Althoff K., Degerman J., Olsson T., Thorlin T., Eriksson P., Time-Lapse Microscopy and Image Processing for Stem Cell Research - Modeling Cell Migration, Proceedings of SPIE, Vol. X, San Diego, USA, February 15-20, 2003.

40.Gustavsson T., Althoff K., Degerman J., Olsson T., Thoreson A.C., Thorlin T., Eriksson P., Time-Lapse Microscopy and Hidden Markov Modeling for Applications to Stem Cell Research. The $20^{\text {th }}$ Annual Houston Conference on Biomedical Engineering Research, Houston, USA, April 2003.

41.Lindblad J, Wählby C, Bengtsson E, Zaltsman A. Image Analysis for Automatic Segmentation of Cytoplasms and Classification of Racl Activation. Submitted for journal publication, 2003, also in $\mathrm{PhD}$ thesis of J Lindblad, Uppsala Univ.2003.

42.Wählby C, Lindblad J, Vondrus M, Bengtsson E, Björkesten L. Algorithms for cytoplasm segmentation of fluorescence labelled cells. Analytical Cellular Path 24:101-111, 2002.

43.Nilsson, M. et al. Padlock probes: Circularizing oligonucleotides for localized DNA detection. Science 265, 2085-2088 (1994).

44.Nilsson, M., Barbany, G., Antson, D.-O., Gertow, K. \& Landegren, U. Enhanced detection and distinction of RNA by enzymatic probe ligation. Nat. Biotechnol. 18, 791-793 (2000). 